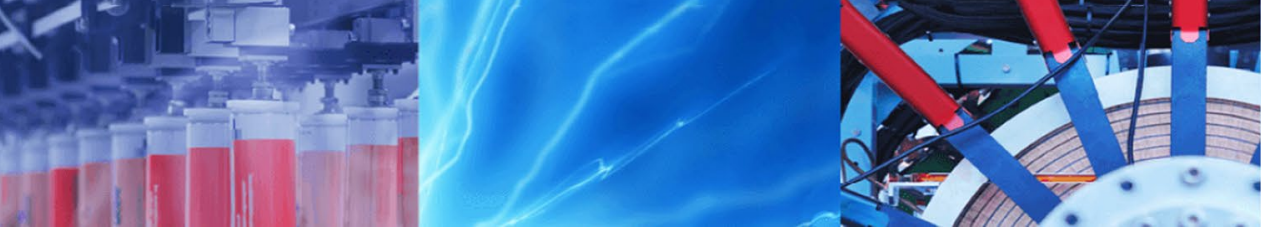

Research Article

\title{
Enhancement in photoluminescence properties of organic compound PS/PPO by cerium fluoride nanoparticles doping
}

\author{
M. Karimi ${ }^{1}$ - M. Raeisi ${ }^{1} \cdot$ M. Bagherzadeh ${ }^{2}\left(D \cdot\right.$ F. Payami $^{2}$
}

(c) Springer Nature Switzerland AG 2019

\begin{abstract}
Herein, a new polymeric nanocomposite of 2,5-diphenyloxazole (PPO) and polystyrene (PS) doped with cerium fluoride $\left(\mathrm{CeF}_{3}\right)$ nanoparticles (PS/PPO/CeF 3$)$ was prepared, characterized and its photoluminescence property was reported for the first time. The PS as a base matrix and PPO as a fluor were chose for a scintillator solution substrate and $\mathrm{CeF}_{3}$ nanoparticles dispersed into it to construct a new nanocomposite scintillator. The PS liquid polymeric matrix with $0.5 \mathrm{wt} \%$ of PPO was loaded with different percentage of $\mathrm{CeF}_{3}$ nanoparticles. Structural characterizations showed successful preparation of nanocomposites and a mean size of $\mathrm{CeF}_{3}$ nanoparticles as $(13 \pm 3) \mathrm{nm}$ in the base polymer matrix. The measured photoluminescence showed an enhancement about 3 times in comparison to PS/PPO composite when 10\% of $\mathrm{CeF}_{3}$ nanoparticles doped into the polymeric composite matrix under UV excitation. This effect is due to fluorescence resonance energy transfer in $\mathrm{Ce}^{3+}$ ions ( $5 d \rightarrow 4 f$ transition energy) to the $\mathrm{PPO}$ within the $\mathrm{PS} / \mathrm{PPO} / \mathrm{CeF}_{3}$ nanocomposite. Observed results were presented and discussed.
\end{abstract}

Keywords Nanoparticles $\cdot \mathrm{CeF}_{3} \cdot \mathrm{PS} / \mathrm{PPO} / \mathrm{CeF}_{3} \cdot$ Nanocomposite $\cdot$ Luminescence $\cdot$ Scintillator $\cdot$ Photoluminescence

\section{Introduction}

Convectional organic scintillators due to fast time response, easy fabrication and low cost are widely used in some sector of fundamental and applied field. Because of their low stopping power, they are unsuitable for high energy $X$ and gamma radiation $[1,2]$. In the last two decades new composites of polymer base organic scintillators doped by inorganic nanoparticles were suggested [3-9]. The transfer energy referred as forester mechanism could lead to the enhancement of composites luminescence.

Early organic-inorganic scintillator composites were made used a grinding method, but their transparency was low [3]. This problem can be solve by using small size of nanoparticles as a filler via increasing the index matching of the components [4]. For example, Kang et al. [5] matched index of nanoparticles and the polymer matrix and reported some nanocomposites with high transparency. Cai et al. [6] reported on luminescence quenching of the polymer due to the charge transfer between the polymer and $\mathrm{Bil}_{3}$ in a conjugated polymer composite with $\mathrm{Bil}_{3}$. Oleic acid as a matrix was loaded with high nanoparticle doping while keeping the transparency up to $70 \%$ [7]. Feller et al. [8] incorporated non-fluorescent $\mathrm{Gd}_{2} \mathrm{O}_{3}$ nanocrystals in polyvinyl toluene (PVT) matrix and reported an enhancement in its luminescence due to fluorescence resonance energy transfer (FRET) from PVT to $\mathrm{Gd}_{2} \mathrm{O}_{3}$ was reported.

$\mathrm{CeF}_{3}$ nanoparticles due to the allowed $5 d-4 f$ transition in $\mathrm{Ce}^{3+}$ ion have significant fluorescence properties, high density, short decay time, fast response and high resistance to radiation. Due to $5 d-4 f$ transition in $\mathrm{Ce}^{3+}$ ions, the doped $\mathrm{Ce}^{3+}$ ions are good candidate materials for fast and efficient scintillators. However, most of the $\mathrm{Ce}^{3+}$ ions

$\triangle$ M. Raeisi, raeisi.morteza@gmail.com; $₫$ M. Bagherzadeh, mjmo123@yahoo.com | 'Department of Physics, Faculty of Science, Shahrekord University, Shahrekord, Islamic Republic of Iran. ${ }^{2}$ Reactor and Nuclear Safety School, Nuclear Science and Technology Research Institute, Isfahan, Islamic Republic of Iran.

SN Applied Sciences (2019) 1:635 | https://doi.org/10.1007/s42452-019-0658-2 
emission is within the UV range which could not directly detect by photodetecors. Therefore to transfer the emission energy from $\mathrm{CeF}_{3}$ to higher wavelengths, a wavelength shifter is needed. Sahi et al. [9] used $\mathrm{CeF}_{3}$ nanoparticles dispersed into PVT matrix with small amount of 2,5 diphenyloxazol (PPO) as a shifter wavelength. A luminescence enhancing is observed under UV excitation to 3 time due to FRET from $\mathrm{CeF}_{3}$ to PPO.

Due to weak fluorescence efficiency and short optical attenuation length of polymers, they cannot be an effective scintillator. But their role as transfer energy of incident energy to flour is important [10], and the most of plastic scintillators is PVT, PPO and polystyrene (PS) polymers matrix. In this research, due to low cost and availability of PS we chosen it as a scintillator solution substrate. The $\mathrm{CeF}_{3}$ nanoparticles synthesized and dispersed into the PS matrix in conjunction with PPO to construct a new nanocomposite scintillator. The luminescence of PPO was enhanced under UV excitation.

\section{Experimental}

\subsection{Materials}

Polystyrene (PS), 2,5-diphenyloxazole (PPO), sodium fluoride ( $\mathrm{NaF}$ ) and cerium (III) nitrate hexahydrate $\left(\mathrm{Ce}\left(\mathrm{NO}_{3}\right)_{3} \cdot 6 \mathrm{H}_{2} \mathrm{O}\right)$ with high purity were purchased from Sigma-Aldrich.

\subsection{Preparation of $\mathrm{CeF}_{3}$ nanoparticles}

Precipitation reaction method was used to synthesize the $\mathrm{CeF}_{3}$ nanoparticles [9]. Briefly, $80 \mathrm{ml}$ of $\mathrm{NaF}, 30 \mathrm{mmol}$, purged with argon gas and heated at $80^{\circ} \mathrm{C}$ under vigorous stirring. After that $60 \mathrm{ml}$ of $\mathrm{Ce}\left(\mathrm{NO}_{3}\right)_{3} \cdot 6 \mathrm{H}_{2} \mathrm{O}, 10 \mathrm{mmol}$, added into the above solution dropwise and the temperature was kept at $80^{\circ} \mathrm{C}$ for $4 \mathrm{~h}$. The resulting $\mathrm{CeF}_{3}$ precipitates were centrifuged and separated from the solution and then washed with deionized water, three times, and finally dried at $50^{\circ} \mathrm{C}$.

\subsection{Preparation of $\mathrm{PS} / \mathrm{PPO} / \mathrm{CeF}_{3}$ nanocomposite}

Firstly the PS/PPO with $0.5 \mathrm{wt} \%$ of PPO, $1.99 \mathrm{~g}$ of PS and $0.01 \mathrm{~g}$ of PPO were dissolved in $10 \mathrm{ml}$ cyclohexane as solvent. Then solution was stirred for $24 \mathrm{~h}$ at room temperature. Then different amount of prepared $\mathrm{CeF}_{3}$ nanoparticles, were added into the above mixture and vigorous stirred for $24 \mathrm{~h}$ at room temperature. Finally, the PS/ $\mathrm{PPO} / \mathrm{CeF}_{3}$ nanocomposite scintillator with $0.5 \mathrm{wt} \%$ of $\mathrm{PPO}$ and different amount (wt\%) of $\mathrm{CeF}_{3}$ nanoparticles were prepared.

\subsection{Instrumentation}

Crystalline structural, size and shape of the nanocomposites were studied by X-ray diffraction (XRD) using a Siemens D500 diffractometer with $\mathrm{Cu} K_{\mathrm{a}}$ (operating at $40 \mathrm{kV}$ and $30 \mathrm{~mA}$ ), scanning electron microscope (SEM) and transmission electron microscopy (TEM) operating at $200 \mathrm{kV}$, respectively. Energy-dispersive $\mathrm{X}$-ray analysis (EDAXA) coupled with SEM was used for the semi-quantities investigation of the nanocomposites. The absorption spectra (UV-Vis) of samples dispersed in methanol were measured by using a spectrophotometer (SHIMADZU UV-3450). A SHIMADZU RF-5301 PC spectrofluorometer was used for recording the photoluminescence emission $(\mathrm{PL})$ spectrums. All the measurements were carried out at room temperature.

\section{Results and discussion}

\subsection{Characterization of prepared nanocomposites}

XRD patterns of the prepared $\mathrm{CeF}_{3}$ nanoparticles are shown in Fig. 1. In the range of $10^{\circ}-70^{\circ}$, the observed peaks at $2 \theta$ positions of $24^{\circ}, 28^{\circ}, 35^{\circ}, 44^{\circ}, 45^{\circ}, 51^{\circ}, 53^{\circ}$ and $65^{\circ}$ are corresponding to space indexes as (110), (111), (112), (333), (113), (302), (221) and (223), respectively. This peak positions are agree with the data reported for $\mathrm{CeF}_{3}$ crystal (JCPDS No. 08-0045) and confirmed hexagonal phase of $\mathrm{CeF}_{3}$ crystals [9]. And also, it is demonstrated that no impurity peaks are detected and the high purity $\mathrm{CeF}_{3}$ is synthesized. With assumption spherical shape for nanoparticles, a particle diameter of about $10 \mathrm{~nm}$ was calculated according to Scherrer's formula from the peak widths. This nanometer size of the prepared $\mathrm{CeF}_{3}$ particles allow us to uniform dispersion of them in the polymer matrixes.

The morphology and size of as-prepared $\mathrm{CeF}_{3}$ nanoparticles and $\mathrm{PS} / \mathrm{PPO} / \mathrm{CeF}_{3}$ nanocomposite were further examined by SEM and TEM (Figs. 2, 3). Figure 2 shows a typical SEM images of $\mathrm{CeF}_{3}$ nanoparticles (Fig. 2a) and $\mathrm{PS} / \mathrm{PPO} / \mathrm{CeF}_{3}$ nanocomposite (Fig. 2b). As can be seen from the SEM image of Fig. 2a, morphology of bulk $\mathrm{CeF}_{3}$ nanoparticles is irregular but their distribution is uniform. The strong peaks just for $\mathrm{Ce}$ and $\mathrm{F}$ atoms were observed in the corresponded EDXA spectrum (Fig. 2c) with atomic ratio as 1:2.8, which can match with $\mathrm{CeF}_{3}$ and showed there is not any impurity in the synthesized $\mathrm{CeF}_{3}$ nanoparticles. As can be seen from the SEM image of Fig. 2b, the $\mathrm{CeF}_{3}$ nanoparticles are well dispersed within the polymer matrix and it also confirms the 
Fig. 1 The XRD pattern of synthesized $\mathrm{CeF}_{3}$ nanoparticles
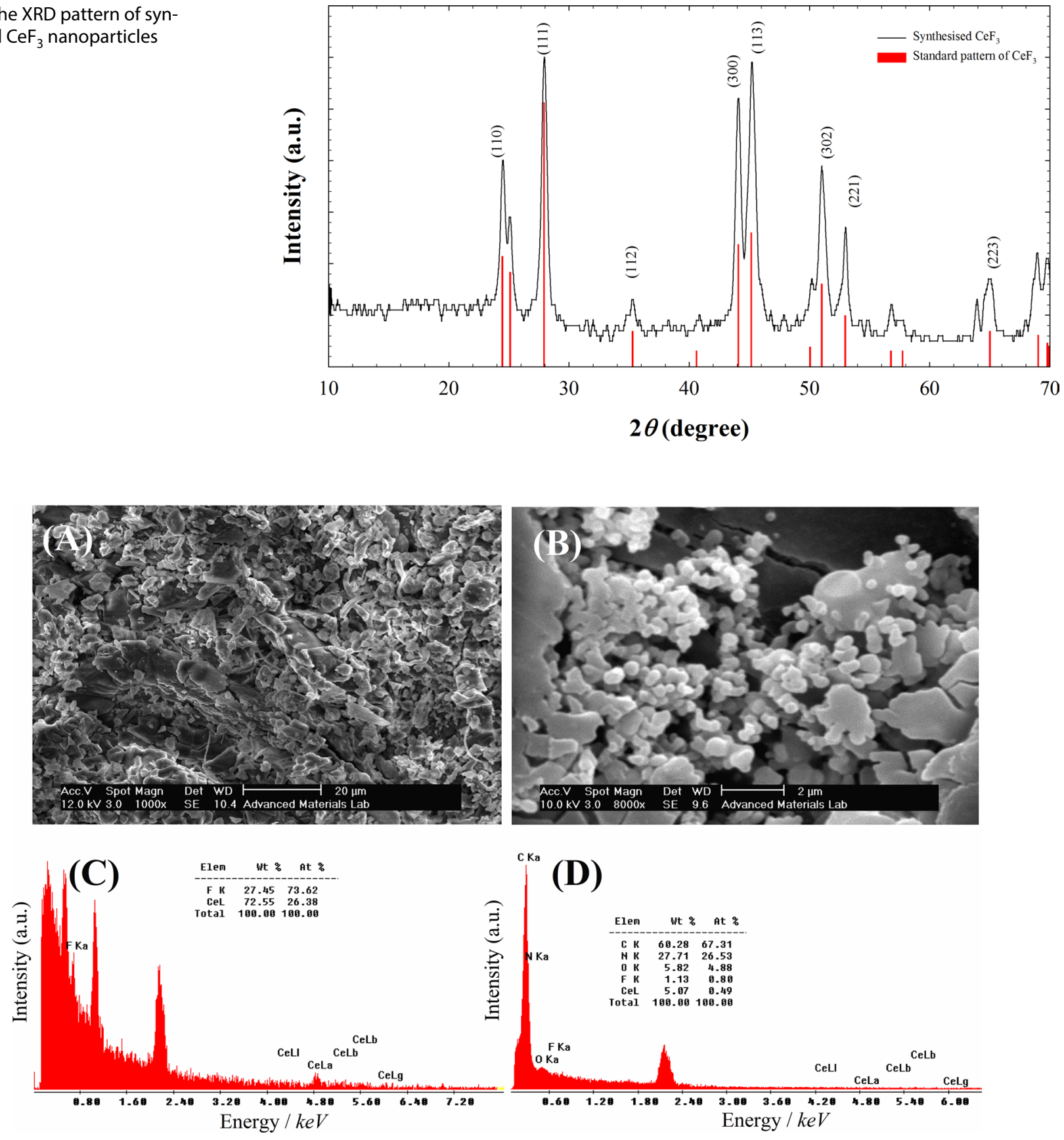

Fig. 2 The typical SEM images $(\mathbf{a}, \mathbf{b})$ and corresponded EDXA spectrum $(\mathbf{c}, \mathbf{d})$ of $\mathrm{CeF}_{3}$ nanoparticles $(\mathbf{a}, \mathbf{c})$ and $\mathrm{PS} / \mathrm{PPO} / \mathrm{CeF}_{3}$ nanocomposite $(\mathbf{b}, \mathbf{d})$

correct way of making polymer nanocomposite in this study. In the corresponded EDXA spectrum of PS/PPO/ $\mathrm{CeF}_{3}$ nanocomposite (Fig. $2 \mathrm{~d}$ ) the strong peaks of $\mathrm{Ce}$ and $\mathrm{F}$ atoms are still presented along with corresponded polymer elements, $\mathrm{C}, \mathrm{O}$ and $\mathrm{N}$ atoms. Observed images and EDXA results successfully confirmed preparation of $\mathrm{CeF}_{3}$ nanoparticles with high purity and uniform dispersion of them into the polymer matrix.
The structural size of the prepared nanomaterials were calculated by TEM. Figure 3 shows the typical images of $\mathrm{CeF}_{3}$ nanoparticles (Fig. 3a), PS/PPO/CeF ${ }_{3}$ nanocomposite (Fig. 3b) and related distributions size of them (Fig. 3c, d). As displayed in Fig. $3 \mathrm{a}$, the $\mathrm{CeF}_{3}$ nanoparticles exhibited spherical morphology and its agglomeration is small. Their distribution is shown in Fig. $3 \mathrm{c}$ that indicates the main average size of prepared $\mathrm{CeF}_{3}$ nanoparticles $(13 \pm 3 \mathrm{~nm})$ 
Fig. 3 The TEM images $(\mathbf{a}, \mathbf{b})$ and its distribution size $(\mathbf{c}, \mathbf{d})$ of the $\mathrm{CeF}_{3}$ nanoparticles (a, c) and the PS/PPO/CeF 3 nanocomposite $(\mathbf{b}, \mathbf{d})$
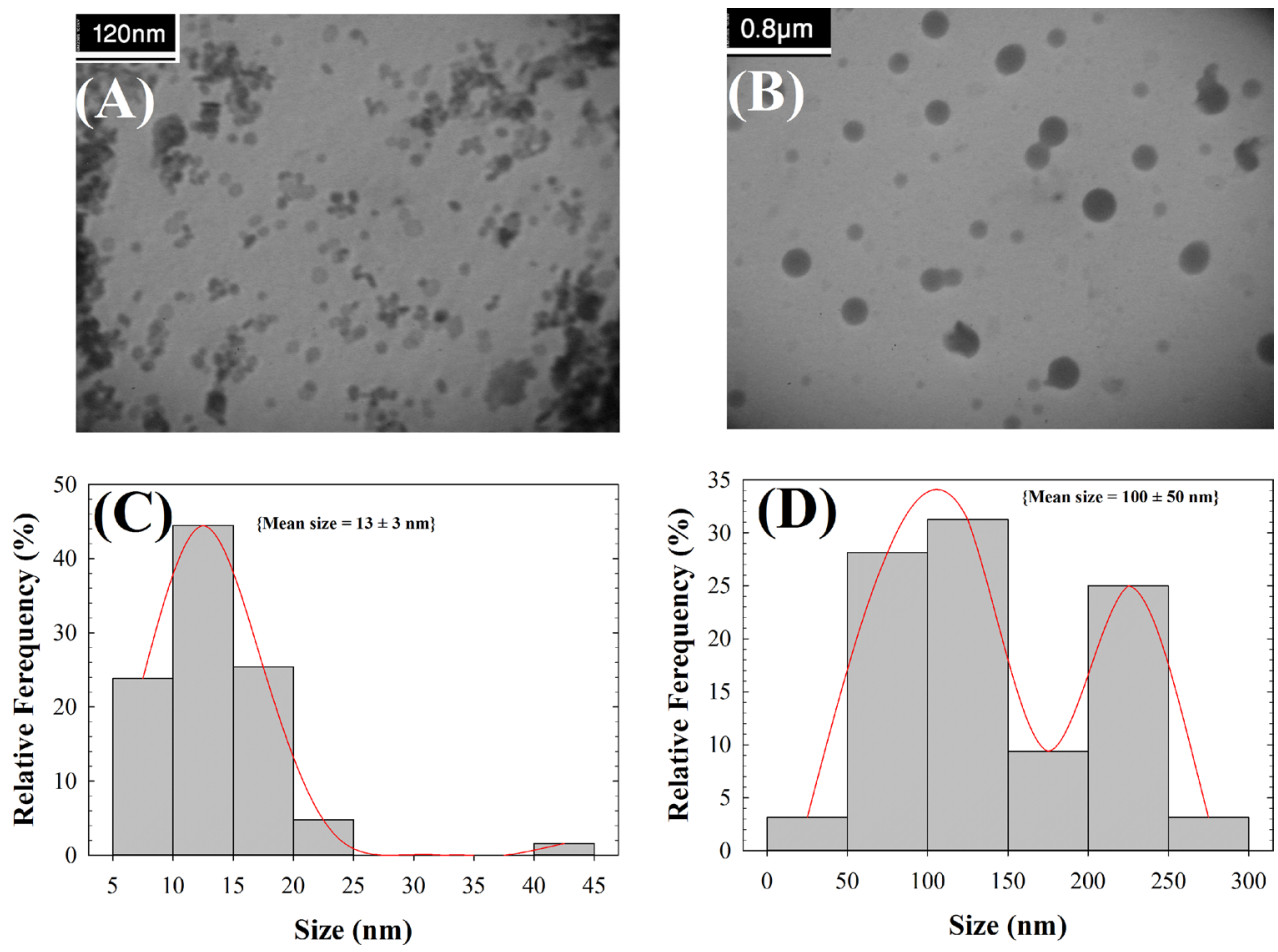

is in good agreement with the result of the XRD pattern. The typical TEM image of PS/PPO/CeF ${ }_{3}$ nanocomposite is shown in Fig. 3b. As can be seen $\mathrm{CeF}_{3}$ nanoparticles uniformly dispersed in the polymer matrix with totally spherical shape. But, the size of the spherical $\mathrm{CeF}_{3}$ nanoparticles increased during dispersion in the polymer matrix and an agglomeration was occurred during synthesize of the PS/ $\mathrm{PPO} / \mathrm{CeF}_{3}$ nanocomposite. However, the observed uniform nanocomposite contains the $\mathrm{CeF}_{3}$ with the mean size as $100 \pm 50 \mathrm{~nm}$ (Fig. 3d).

\subsection{Photoluminescence study of the nanocomposites}

The photoluminescence of the composition of components for construction of the $\mathrm{PS} / \mathrm{PPO} / \mathrm{CeF}_{3}$ nanocomposite is studied and compared together to show the observed enhancements. The photoluminescence (PL) emissions of PS and PPO, along with absorption spectrum of PPO measured at room temperature is shown in Fig. 4. As can be seen from Fig. 4, the existing overlap between the PPO absorption and PS emission spectrums is adequate to transfer energy between them. Consequently, more emission will be observed by PPO at longer wavelength.

The absorption and PL emission spectrum of PS/PPO, along with $\mathrm{PL}$ emission spectrum of $\mathrm{CeF}_{3}$ nanoparticles are presented in Fig. 5. As can be seen from Fig. 5, there is an overlaps between the emission of $\mathrm{CeF}_{3}$ and absorption of PS/PPO that in turn accomplish the condition for FRET.

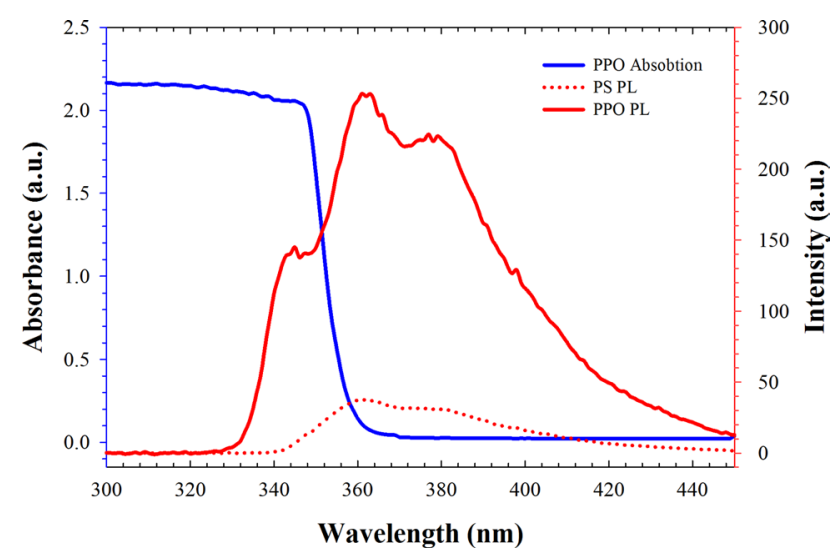

Fig. 4 The absorption spectrum of PPO (blue line) and PL spectrums (red lines) of PPO (solid line) and PS (dotted line) in cyclohexane at $2 \mathrm{~g} / \mathrm{l}$ of them

Thereupon energy can be transferred from $\mathrm{CeF}_{3}$ nanoparticles to PS/PPO. In addition, the lifetime of $\mathrm{CeF}_{3}$ (30 ns) $[11,12]$ is sufficiently longer that of PPO (1.6 ns) [13] satisfy FRET condition to occur.

Different percentage of $\mathrm{CeF}_{3}$ nanoparticles were added into the PS matrix that contains constant percentage of PPO, $0.5 \mathrm{wt} \%$, to proof enhancement effect of $\mathrm{CeF}_{3}$ nanoparticles on the emission of PS/PPO. Figure 6 shows the $\mathrm{PL}$ spectrum of excited $\mathrm{PS} / \mathrm{PPO} / \mathrm{CeF}_{3}$ nanocomposites at $290 \mathrm{~nm}$. As can be seen the photoluminescence of PS/PPO composites notably enhanced by the $\mathrm{CeF}_{3}$ nanoparticles. 


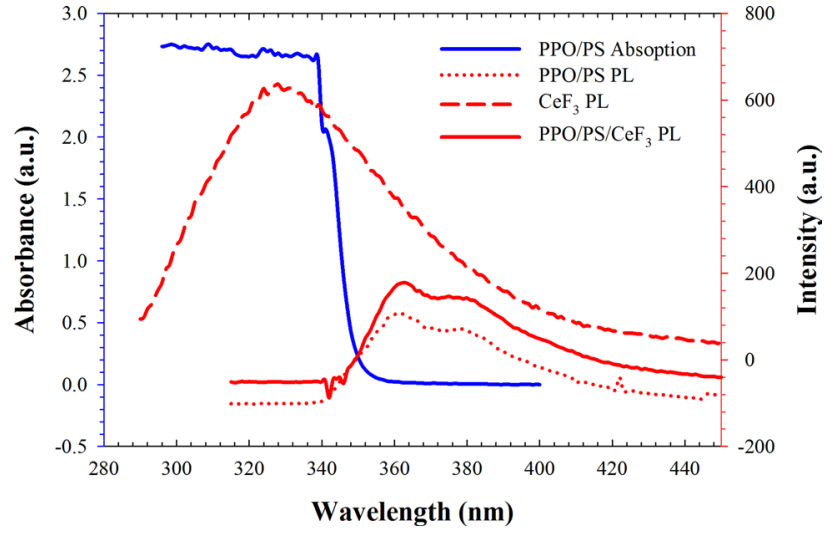

Fig. 5 The absorption spectrum of PS/PPO (blue line) and PL spectrums (red lines) of PS/PPO (dotted line), $\mathrm{CeF}_{3}$ nanoparticles (dashed line), and PS/PPO/CeF 3 (5 wt $\% \mathrm{CeF}_{3}$ ) nanocomposite (solid line) in cyclohexane at $2 \mathrm{~g} / \mathrm{l}$ of them

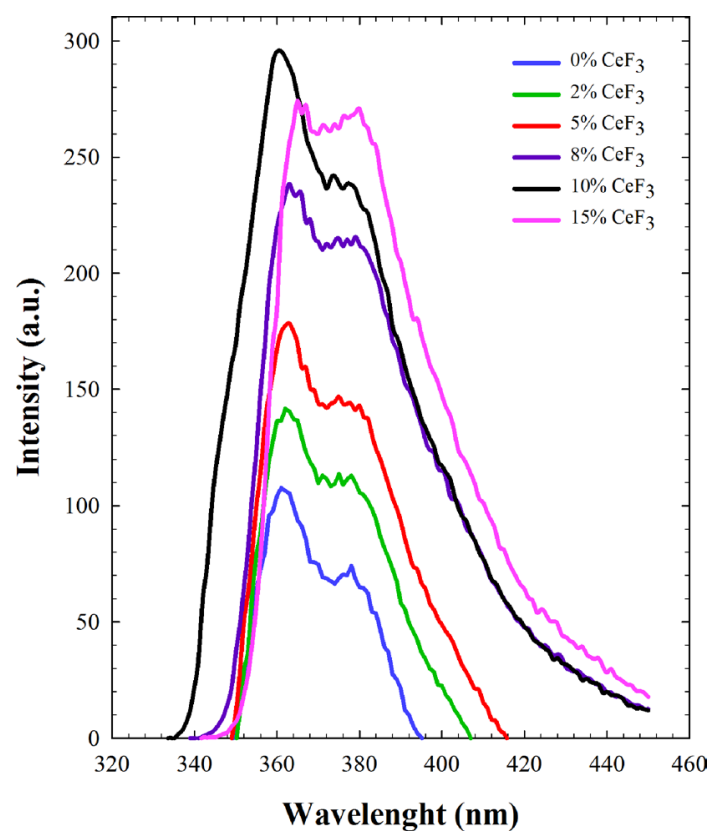

Fig. 6 The PL spectrums of PS/PPO/CeF 3 nanocomposites with different loading percentage of $\mathrm{CeF}_{3}$ nanoparticles

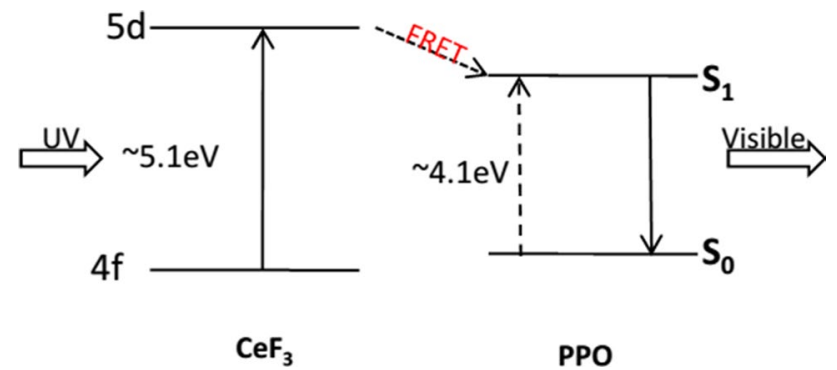

Fig. 7 Diagram of fluorescence resonance energy transfer (FRET) from $\mathrm{CeF}_{3}$ to PPO
As expected, due to the energy transfer from $\mathrm{CeF}_{3}$ nanoparticles to the PPO in the PS matrix as shown in Fig. 7, an enhancement in the emission intensity of nanocomposite are presented. Furthermore, absence of $\mathrm{CeF}_{3}$ nanoparticles emission in the spectrums, confirm the FRET phenomenon occurred between $\mathrm{CeF}_{3}$ nanoparticles and PS/PPO composite. Also, as can be seen from Fig. 6 , an enhancement about 3 times was observed in the emission of nanocomposites up to $10 \mathrm{wt} \%$ of $\mathrm{CeF}_{3}$ doping, and then reduced to less than 1.5 times for $15 \mathrm{wt} \%$ of $\mathrm{CeF}_{3}$. It is clear that increasing in percentage of $\mathrm{CeF}_{3}$ in the nanocomposite, cause to a red shift in the emission of nanocomposites.

\section{Conclusion}

$\mathrm{PS} / \mathrm{PPO} / \mathrm{CeF}_{3}$ nanocomposite was prepared with $0.5 \mathrm{wt} \%$ $\mathrm{PPO}$ and various percentage of $\mathrm{CeF}_{3}$ nanoparticles. Its structure and luminescence properties of constituents were studied systematically. Our results showed an enhanced luminescence in PS/PPO when embedded with $\mathrm{CeF}_{3}$ nanoparticles. Enhancement in the luminescence of PS/PPO scintillator reaches about 3 times when doped with $10 \mathrm{wt} \%$ of $\mathrm{CeF}_{3}$ nanoparticles. This enhancement can be assigned to the energy transfer, the increase of the stopping power by doping $\mathrm{CeF}_{3}$ nanoparticles. However, our findings represent a new method to improve PS/PPO organic scintillators for radiation detection.

Acknowledgements The facilities of this work were supported by NSTRI and University of Shahrekord, and the authors are gratefully acknowledged.

\section{Compliance with ethical standards}

Conflict of interest The authors declare that they have no conflict of interest.

\section{References}

1. Knoll GF (2010) Radiation detection and measurement. Wiley, Hoboken

2. Milbrath BD, Peurrung AJ, Bliss $M$, Weber WJ (2008) Radiation detector materials: an overview. J Mater Res 23(10):2561-2581

3. Vasil'chenko V, Solov'Ev A (2003) Properties of composite scintillators in static and dynamic states. Instrum Exp Tech 46(6):758-764

4. McKigney EA, Del Sesto RE, Jacobsohn LG, Santi PA, Muenchausen RE, Ott KC, McCleskey TM, Bennett BL, Smith JF, Cooke DW (2007) Nanocomposite scintillators for radiation detection and nuclear spectroscopy. Nucl Instrum Methods Phys Res Sect A 579(1):15-18

5. Kang Z, Barta M, Nadler J, Wagner B, Rosson R, Kahn B (2011) Synthesis of $\mathrm{BaF}_{2}$ : Ce nanophosphor and epoxy encapsulated transparent nanocomposite. J Lumin 131(10):2140-2143 
6. Cai W, Chen Q, Cherepy N, Dooraghi A, Kishpaugh D, Chatziioannou A, Payne S, Xiang W, Pei Q (2013) Synthesis of bulk-size transparent gadolinium oxide-polymer nanocomposites for gamma ray spectroscopy. J Mater Chem C 1(10):1970-1976

7. Zhong H, Zhao Y, Li Y, Pei Q (2008) Photoluminescence quenching of conjugated polymer nanocomposites for gamma ray detection. Nanotechnology 19(50):505503

8. Feller RK, Purdy GM, Ortiz-Acosta D, Stange S, Li A, McKigney EA, Esch El, Muenchausen RE, Gilbertson R, Bacrania M (2011) Large-scale synthesis of CexLa1-xF3 nanocomposite scintillator materials. J Mater Chem 21(15):5716-5722

9. Sahi S, Chen W, Jiang K (2015) Luminescence enhancement of PPO/PVT scintillators by $\mathrm{CeF}_{3}$ nanoparticles. J Lumin 159:105-109

10. Nurmukhametov R, Volkova L, Kabanov S (2006) Fluorescence and absorption of polystyrene exposed to UV laser radiation. J Appl Spectrosc 73(1):55-60
11. Moses W, Derenzo S, Weber M, Ray-Chaudhuri A, Cerrina F (1994) Scintillation mechanisms in cerium fluoride. J Lumin 59(1-2):89-100

12. Moses W, Derenzo SE (1989) Cerium fluoride, a new fast, heavy scintillator. IEEE Trans Nucl Sci 36(1):173-176

13. Luchowski R (2011) Two-photon excitation of 2,5-diphenyloxazole using a low power green solid state laser. Chem Phys Lett 501(4-6):572-574

Publisher's Note Springer Nature remains neutral with regard to jurisdictional claims in published maps and institutional affiliations. 